\title{
Scaling up Distributed Massive MIMO: Why and How
}

\author{
Cheng-Ming Chen, Andrea P. Guevara, and Sofie Pollin \\ Department of Electrical Engineering, KU Leuven, Belgium \\ Email:\{cchen,aguevara,spollin\}@esat.kuleuven.be
}

\begin{abstract}
Massive MIMO (MaMIMO) is a technology of primary interest for sub-6 GHz operation in the next generation cellular systems. While MaMIMO is most often linked to macrocell scenarios, where a single cell serves many users distributed over a large area, network densification will also result in scenarios where many users are served by a MaMIMO base station (BS) that is nearby. A key question is how to scale up MaMIMO: should we add more antennas to a given cell, or create multiple smaller and distributed cells that can cooperate? This paper documents the measured performance of a very dense MaMIMO system for an indoor-to-outdoor propagation environment. The impact of the number of antennas, and the distribution of the antenna elements is experimentally verified for a simplified linear deployment of the BSs. Concretely, we serve 12 closely located users with 16, 32 or 64 antennas. We compare a centrally positioned collocated array and two distributed arrays with their uplink throughput in a licensed $2.6 \mathrm{GHz}$ band. The experimental results show that 12 users can be served with only 32 antennas for the distributed topology, which is effectively only 16 antennas per MaMIMO BS. For the specific case analyzed in our measurement campaign, with the centralized deployment, 64 antennas are needed to obtain good performance, while distributing the antenna elements in two sub-arrays improves total performance and fairness between the users.
\end{abstract}

Index Terms-Distributed antenna array, large-scale antenna systems, massive MIMO, software defined radio, spatial separation.

\section{INTRODUCTION}

Massive MIMO (MaMIMO) is considered an important technology for $5 \mathrm{G}$ because it enables two desirable benefits, namely, excellent spectral efficiency and superior energy efficiency [1]. The main concept is to use large antenna arrays at the base stations (BSs) to simultaneously serve many user equipments (UEs). Due to the excess amount of antennas in the BS compared to the number of served UEs, this extra degree of freedom enables simple linear processing in the BS. Additionally, it enables low complexity, single antenna, and energy efficient UEs.

A large number of MaMIMO experiments have been reported in literature, focusing on the measurement and analysis of the channel, to prove the benefits of using large antenna arrays. In [2], the researchers validated the channel characteristics of two kinds of 128-antenna arrays, cylindrical and virtual linear arrays. This work focused on an outdoor environment, where the BS is located on a high building, serving multiple users on the ground. The experiments confirm that MaMIMO achieves a performance close to that of commonly used independent and identically distributed (i.i.d.) Rayleigh chan- nels. Both [3], [4] consider the user separation capability of MaMIMO when increasing the number of antennas in the BS array. With a collocated antenna array (CAA), improvements in user separation saturate when a critical number of antennas is achieved, and the performance becomes limited by the characteristics of the propagation environment. In [5], experimental results of distributed antenna arrays (DAAs) were first reported and exhibited a much better user separation capability for an indoor line-of-sight (LoS) scenario. An important finding is that if the sub-arrays are distributed parallel to the line connecting the users, the enriched angle of arrivals (AoAs) obtained from the distributed array increase the degrees of freedom in the channel and enhance the user separation. In general, these results confirm that centralized MaMIMO performs well in typical fading scenarios, that benefit from a rich scattering. When the channel degrades to a poor-scattered scenario, the benefits resulting from distribute antenna systems become large.

CAA has the advantage of low backhaul overhead. In contrast, DAAs have the potential of achieving diversity even in LoS channels and offering larger coverage at the cost of backhaul deployment overheads [6]. Cell-free MaMIMO is proposed in [7], by using an advanced backhaul, to provide fairly good service to all users. The idea of cell-free MaMIMO is to distribute multiple single antennas of the BS uniformly in a given area, and when it is used with maximum ratio transmitting (MRT), the backhaul overhead is low as collecting channel state information centrally is not necessary. However, when the number of UEs being serviced in the system increases, zeroforcing $(\mathrm{ZF})$ or minimum mean square error (MMSE) still outperforms MRT with a better capability of mitigating interuser-interference (IUI). To efficiently cancel IUI, reciprocity calibration in time division duplex (TDD) based MaMIMO is essential. Distributing the antennas in a sub-array based topology can increase the robustness of TDD reciprocity calibration in distributed MaMIMO system [8], as intra-array and interarray calibrations can both be enhanced. Meanwhile, this kind of distributed MaMIMO topology reduces the complexity of backhauling.

In this paper, we experimentally verify the benefits of distributed MaMIMO in a sub-array based geometry in NonLoS (NLoS) propagation conditions, including building penetration losses as illustrated in Fig 1. When the densification of MaMIMO cells increases, the question arises wether either the collocated MaMIMO with one large array, or the cooperation 


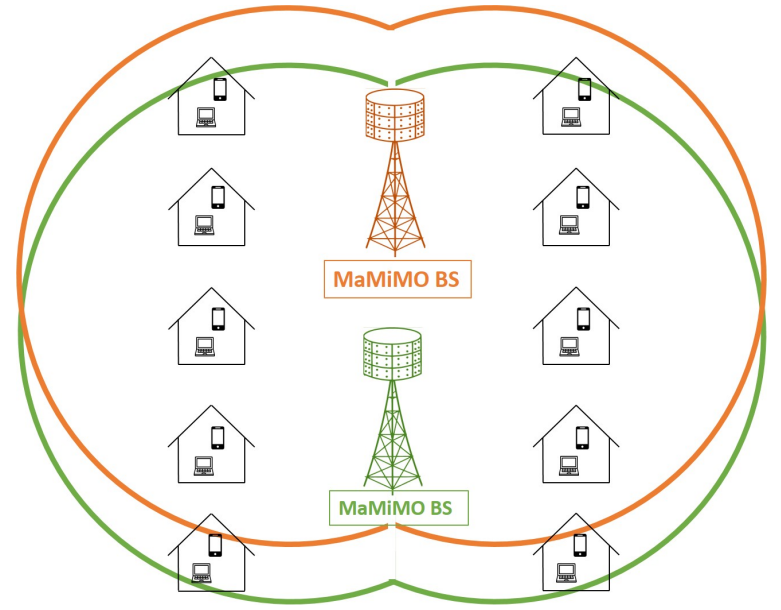

Fig. 1. Distributed MaMIMO, cooperation between multiple cells with a (smaller) MaMIMO array.

between multiple closely located smaller MaMIMO cells, is more beneficial? For a given number of antennas per BS, to what extent can a cooperation between multiple nearby BS increase both the array gain (more effective number of antennas) and the diversity gain?

The paper is organized as follows. The setup of the software defined radio (SDR) testbed for system performance and channel measurement are introduced in section II. Section III describes the system model and the relevant performance metrics. The results of the paper are summarized in section VI and concluded in section V.

\section{EXPERIMENT SETUP}

To emulate a dense MaMIMO network, and evaluate the optimal topology for our specific case study, we measure the UL throughput of $12 \mathrm{UE}$ as function of the number of antennas in combination with the topology of the BS antenna array. Two main configurations are studied: a centralised configuration, and a distributed configuration. In the centralised configuration, two sub-arrays are placed next to each other, in the distributed configuration, two sub-arrays are separated on a line. Below, we detail the scenario set up in our lab, discuss the detailed antenna configuration, and explain some implementation details.

\section{A. Scenario for the Case Study}

An overview of the indoor-to-outdoor scenario that we created in our lab is shown in Fig. 2a. We define a symmetric scenario to ensure a fair comparison between distributed and centralised deployments of the sub-array. For practical reasons, the BS with two antenna arrays are located indoors, with the two antenna arrays deployed centralised or distributed for 64 , 32 and 16 active antennas (Fig. 2b)The relative location of the BS antenna arrays to the respective UEs is important. For the distributed case, our scenario measured a network with two antenna arrays placed $0.9 \mathrm{~m}$ behind two closed windows with insulating glass. The antenna panels are however lower than the windows, so the LoS is also blocked by a wall and metal heating system in the distributed case, despite the presence of the windows. The metal heating system is denoted by a grey rectangles in Fig. 2b. The two arrays are $6.35 \mathrm{~m}$ apart.

For the centralised deployment, the two arrays are placed in the center of the indoor corridor; just before the antenna array, there is a shelf as represented by the black rectangle. In both cases, the two arrays are connected to two clusters with National Instrument universal software radio peripherals (USRPs) which run the LabVIEW Communications MIMO Application Framework. ${ }^{1}$ The UEs in this experiment are implemented with six USRPs, each of them with two dipole antennas, for a total of 12 streams. These 12 streams could represent 12 UEs. This means that UEs are always grouped in a pair with a distance of only $20 \mathrm{~cm}$, as they are bundled in the same USRP. All UEs are located quite closely, i.e., the distance between the furthest UEs is only $9.2 \mathrm{~m}$. The distance of the center of the arrays (for both distributed and collocated) to the closest UE is around $6.5 \mathrm{~m}$. We conducted the experiment in a quasi-stationary environment, meaning no people were walking around during the measurement ${ }^{2}$.

\section{B. Antenna Configuration and Selection}

When studying the impact of the number of antennas for each topology, we need to specify how antennas are selected in our experiments. In addition to the antenna selection topology, the antenna element and array topology has a large impact on the system level performance of MaMIMO. Concretely, each element is a dual band directional antenna and our experiments are conducted at $2.6 \mathrm{GHz}$ with $20 \mathrm{MHz}$ bandwidth. Details of our antenna design can be referred to [10].

We detail the antenna selection strategy per sub-array. Specifically, when the number of antennas scales down from 64 to 32 : In the distributed case, the bottom 16 antennas are selected from each sub-array; while in the collocated case, one full antenna array is disabled, and the other full planar array with 32 antennas is activated. In a similar way, when scaling down to 16 antennas: For the distributed 16-antenna scenario, 8 antenna elements in the left bottom corner of each sub-array are selected; For the collocated case, the bottom 16 antennas from one of the sub-arrays are selected. All antennas at the $\mathrm{BS}$ are co-polarized to the dipoles in the UEs.

\section{Distributed Processing}

The use of USRP's and the NI MaMIMO framework has already been reported in various studies related to centralized MaMIMO [3]. Our setup is very similar to those approaches, only we separated half of the testbed, and half of the USRPs in a second rack that can be distributed to some extent as both racks are connected with optical fibers.

A Time Division Duplex (TDD) based long term evolution (LTE)-like frame structure is adopted in this MaMIMO system. The default frame structure in the baseline implementation we use only uses two OFDM symbols per slot for UL. Given

\footnotetext{
${ }^{1}$ The detailed single cell system architecture can be referred to [9].

${ }^{2}$ For each UE channel vector, we analysed the time correlation function. For the measured time, their normalized correlation is above 0.99.
} 


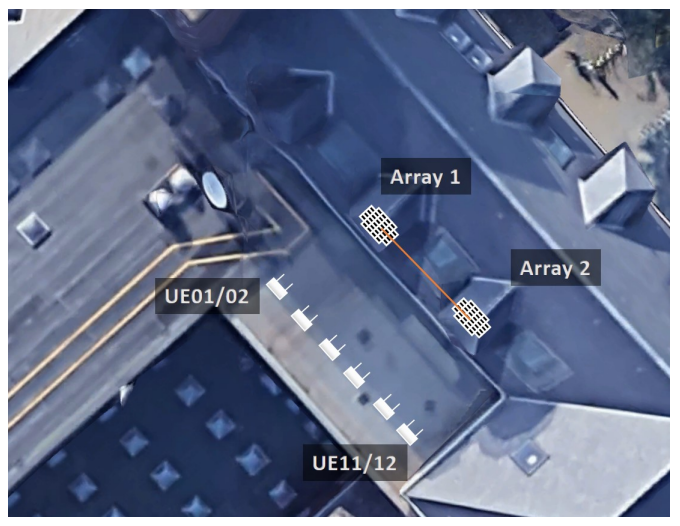

(a) Overlook of the indoor-to-outdoor scenario from the roof of ESAT building of KU Leuven

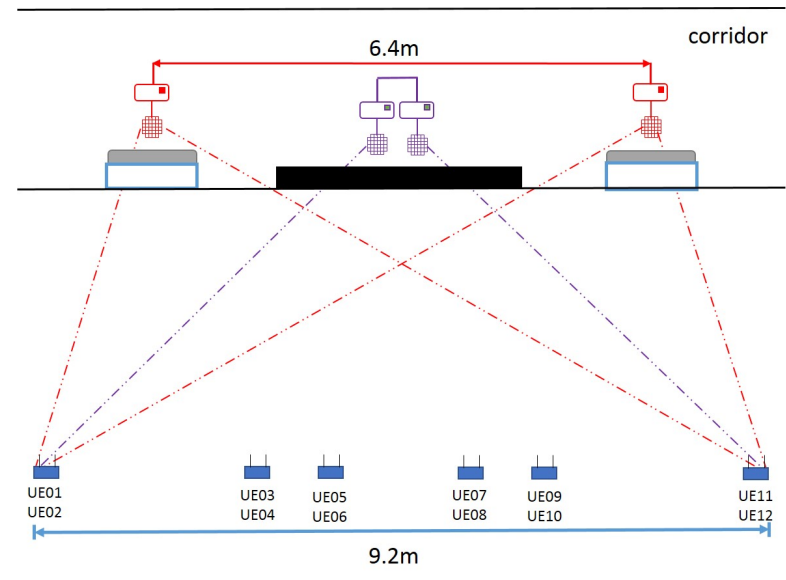

(b) The relative locations of the collocated and distributed antenna arrays to the 12 UEs

Fig. 2. A symmetric indoor-to-outdoor MaMIMO scenario. We compare the combination of antenna topologies and the number of BS antennas to the throughput and channel characteristics of UL from the 12 UEs.

overhead for pilots and the fact that we use uncoded 16 QAM for our measurements, the peak UL overall throughput given this frame structure and constellation is around 218.8 Mbits/sec (Mbps) and 18.23 Mbps per UE.

\section{System Model}

When reporting the measured performance of our distributed MaMIMO system, is it important to specify the exact metrics used for the logging and data analysis. An uplink (UL) single-cell MaMIMO system is considered in this paper, as this allows us a system level throughput comparison of multiple topologies independent of the reciprocity calibration performance. A BS with a large number of antennas $M$ communicates with $K(K \leq M)$ single-antenna UEs. Vector $\mathbf{h}_{k, n, t}$ represents the $k$ th column of the channel matrix $\mathbf{H}_{n, t} \in$ $\mathbb{C}^{M \times K}$ at subcarrier $n$ and snapshot $t$, with $1 \leq n \leq N$ and $1 \leq t \leq T$, where $N$ is the total number of subcarriers, $T$ is the total number of measurement snapshots. We analyze the received power and channel obtained from the UL channel estimation for each configuration. For metrics such as Channel Hardening $(\mathrm{CH})$ and Relative Orthogonality (RO), power normalization is neglected. Therefore, our results clearly show the impact of power variation between the antenna elements in the distributed case. However, while analyzing the Singular Value Spread (SVS), per UE based power normalization should be considered, as was for instance done in [2], [3]:

$$
\mathbf{h}_{k, n, t}^{\text {norm }}=\sqrt{\frac{M N T}{\sum_{n=1}^{N} \sum_{t=1}^{T}\left\|\mathbf{h}_{k, n, t}^{\text {meas }}\right\|_{F}^{2}}} \mathbf{h}_{k, n, t}^{\text {meas }},
$$

where superscript meas represents the measured channel, while norm stands for the normalized channel. For notational simplicity, we ignore the dependence of $\mathbf{H}$ on $n$ and $t$. Let $\mathrm{s} \in \mathbb{C}^{K \times 1}$ be the vector signal transmitted by the $K$ UEs simultaneously to the BS, it satisfies the per-user equal transmit power constraint $\mathbb{E}\left[\left\|s_{k}\right\|^{2}\right]=E_{s}$. The vector of the received signals $\mathbf{y} \in \mathbb{C}^{M \times 1}$ at the $\mathrm{BS}$ is given as $\mathbf{y}=\mathbf{H} \mathbf{s}+\mathbf{n}$, where $\mathbf{n}$ is an i.i.d. circularly-symmetric complex Gaussian distributed noise vector, with the noise-variance $\mathbf{n} \sim \mathcal{C N}\left(\mathbf{0}, N_{o} \mathbf{I}_{K}\right)$.

In addition to measured throughput, using uncoded MMSE decoding, we introduce three metrics to evaluate the orthogonality and inter-user-interference (IUI) of the system, namely, $\mathrm{CH}, \mathrm{RO}$ and SVS.

\section{A. Channel Hardening}

By applying linear maximum ratio combining (MRC) on the received signals from a large number of antennas, $\mathrm{CH}$ is obtained, which represents the fact that the MRC-combined signal is less affected by Rayleigh fading. Here, we analyse the $\mathrm{CH}$ by comparing the channel gain variations from individual single antenna signals and the MRC combined channel gain from all antennas, which can be represented as

$$
C H_{k, m}^{s}=\left|h_{k, m}\right|^{2} \text { and } C H_{k}^{m}=\left\|\mathbf{h}_{k}\right\|_{F}^{2},
$$

respectively; where $(.)_{k, m}$ denotes the $(k, m)_{t h}$ entry of a matrix and superscript $s$ and $m$ denotes single antenna and MRC, respectively. Due to channel hardening, the combined channel $C H_{k}^{m}$ seen by user $k$ should become more stable. It should be noted that there is no normalization for the channel.

\section{B. Relative Orthogonality}

We define RO from the Wishart matrix $\mathbf{W}=\mathbf{H}^{H} \mathbf{H}$, which is a good indication of orthogonality between each pair of UEs:

$$
R O=10 \times \log _{10}\left(\mathbb{E}\left[\frac{a b s(\mathbf{W})}{\max (a b s(\mathbf{W}))}\right]\right),
$$

where $\max ($.$) and a b s($.$) stand for maximum of a matrix and$ element-wise absolute operation, respectively. Please not that $\mathbf{H}$ in this case comes from $\mathbf{H}^{\text {meas }}$. The expectation operation is applied in both frequency and time domain. 


\section{Singular Value Spread}

MaMIMO decorrelates UEs by the orthogonality of their channels. The $K$ singular values $\left(\sigma_{1}, \sigma_{2}, \ldots, \sigma_{K}\right)$ (in descending order) of the channel $\mathbf{H}^{\text {norm }}$ can be used as a measure to quantify orthogonality. Specifically, the ratio between the maximum and minimum singular values are of interest:

$$
\kappa=10 \times \log _{10}\left(\frac{\sigma_{1}}{\sigma_{k}}\right) .
$$

When $\kappa \rightarrow 0, \mathbf{H}^{\text {norm }}$ represents a fully orthogonal channel.

\section{Measurement Results}

In this section, the performance of a collocated and distributed MaMIMO system is compared using the metrics introduced before. Our aim is to carry out a fair comparison, and explain why one array topology is better than the other. For both scenarios, all UEs transmit the same power $20 \mathrm{dBm}$ and all BS antennas have a fixed $37.5 \mathrm{~dB}$ receiver power adjustment. For the channel analysis, we log the estimated channels from one OFDM symbol in one frame, with a sample time of $100 \mathrm{~ms}$ between multiple channel logs. Overall 35 OFDM symbols are collected in the analysis.

\section{A. Power Distribution}

As there is a difference in the pathloss, penetration losses and obstacles seen and antenna pattern for each topology, it is important to verify if the received power distributions from two different topologies are similar. This can be achieved by looking into the received power distribution at BS antennas as shown in Fig. 3. The power per antenna is averaged over 1200 subcarriers and 35 symbols to eliminate fading. If in both topologies, the BS antennas receive similar power levels ${ }^{3}$, the performance will depend on the correlation of the channel matrix. Here we sample the estimated UL channel power from UE2, UE7 and UE12 from all BS antennas. If we look at the power received by the collocated array as in $3 \mathrm{a}$, we notice that there is a big gain variation among the antennas of $15-20 \mathrm{~dB}$, which means that all antennas do not contribute equally even though their pathloss should be very similar. This is consistent to our findings in [10], and the reason is a combination of antenna gain variation and fading (although we averaged here over 35 realisations in 3.5 seconds). Noteworthy, the antenna index in Fig. 3 is counted continuously from the first array to the second array. From Fig. 3b, if we look at the power distribution for UE12, it can be seen that the power of antenna $33-64$ is larger than the power received in antennas 1-32, which is expected from the system topology. In general, the received power levels are quite similar in both topologies.

\section{B. Channel Hardening}

As we measured many channel realisations in both frequency and time domain with 1200 subcarriers and 35 OFDM symbols, we obtain overall 42000 realizations. We first calculate the single antenna channel gain and combined channel

\footnotetext{
${ }^{3}$ This then means that the path losses from different sub-arrays to the UEs are similar.
}
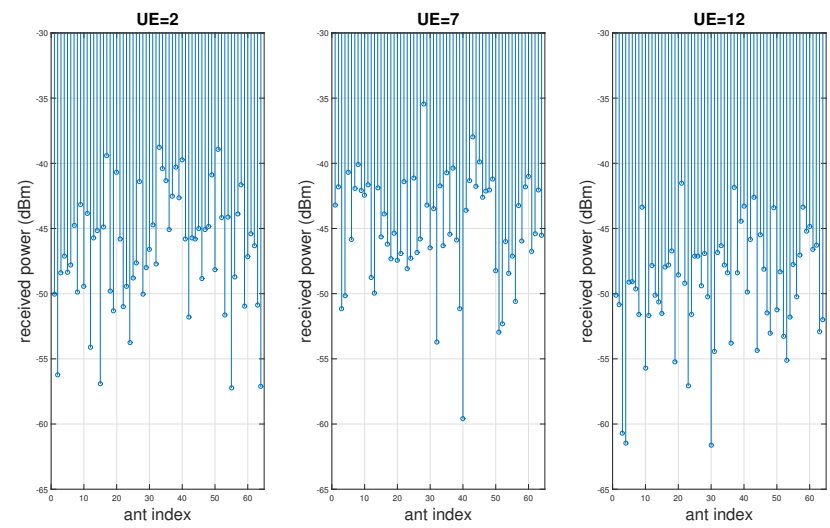

(a) Collocated array
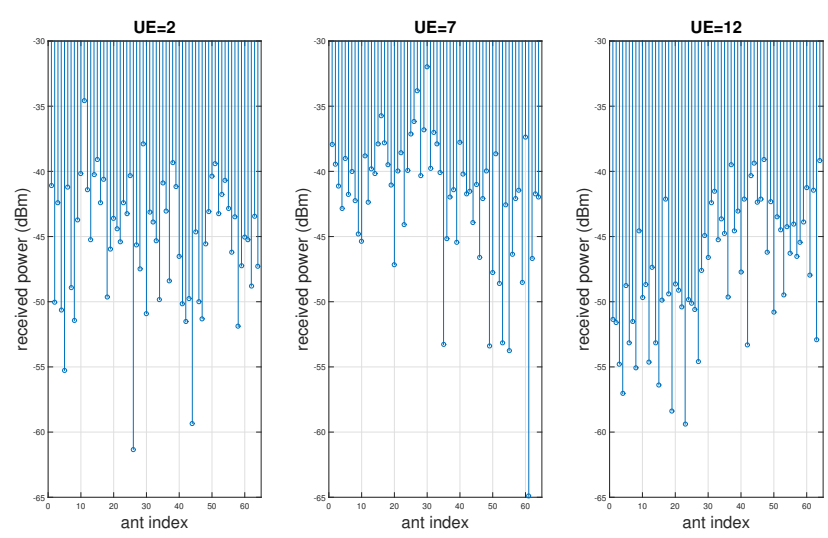

(b) Distributed array

Fig. 3. Received power from UE2, UE 7 and UE12 to collocated and distributed 64-antenna arrays, respectively.

gain with MRC for an increasing array size. These channels are computed for each of the 12 UE. Next, we formulate the variability or fluctuation of the channel gain by computing the inter-quartile ranges (IQR) and median of the distribution of these realizations per UE. Finally, we apply a second IQR and median over median obtained for each UE, and plotted it in Fig. 4. Apparently, with MRC, the median is increased when we compare MRC to a single antenna, this gain is known as array gain. Meanwhile, IQR is also decreased when there are more antennas, this is called the $\mathrm{CH}$ effect, which decreases the variation expected from fading. Interestingly, DAAs have a larger IQR when we treat the antennas individually, and interestingly when we combine all antennas, a DAA scenario results in a slightly lower IQR compared to a CAA scenario. We can interpret this as better user fairness when we distributed the array equally relative to the distribution of UEs. This shows a trend that with more antennas, both centralised and distributed deployments show $\mathrm{CH}$ and array gain improvements. 


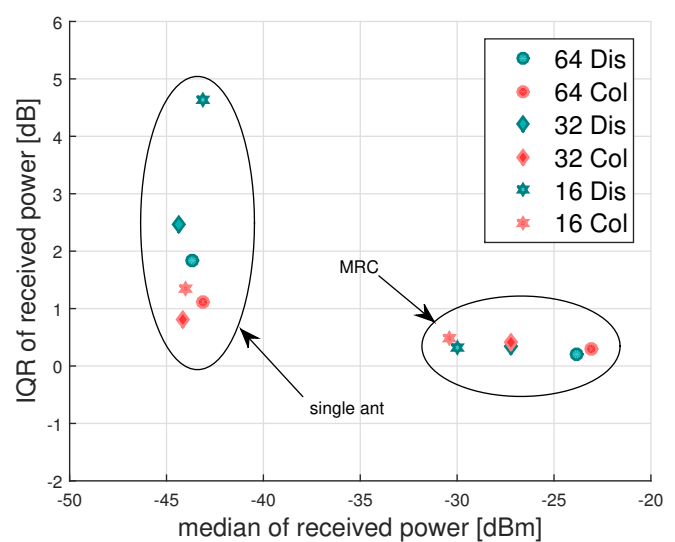

Fig. 4. Median and inter-quartile range (IQR) of the received power in the antenna array. Dis and Col represent distributed and collocated topologies, respectively. The $\mathrm{CH}$ greatly reduces the IQR when relying on MRC.

\begin{tabular}{|l|c|c|c|}
\hline \multicolumn{4}{|c|}{ Overall UL throughput [Mbps] } \\
\hline & 16 antennas & 32 antennas & 64 antennas \\
\hline Collocated & 23.1 & 183.7 & 217.4 \\
\hline Distributed & 25.9 & 205.1 & 218.8 \\
\hline \hline
\end{tabular}

TABLE I. Overall UL throughput w.r.t. different number of antennas

\section{Relative Orthogonality}

Due to channel hardening, the channels become less correlated when then number of antennas increase. The result is a more pronounced diagonal in Fig. 5. For the 64 antennas case, CAA has a stronger diagonal but also has rather strong off-diagonals as compare to DAAs. In general, we see that both DAA and CAA improve the diagonals when increasing the number of antennas, which is as expected.
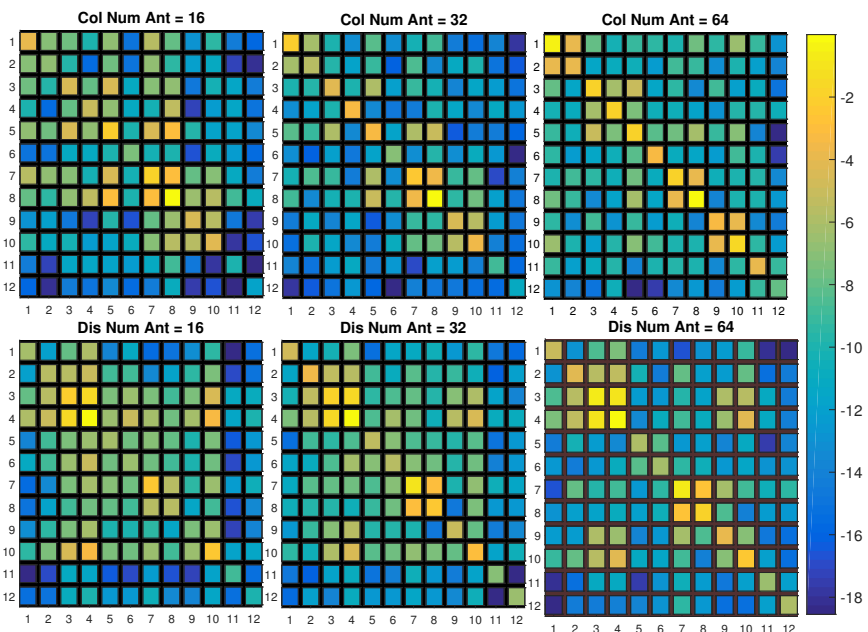

Fig. 5. Comparison of relative orthogonality for both antenna deployments in combination with number of antennas 64, 32 and 16.

\section{Singular Value Spread}

Fig. 6 represents channel orthogonality as a cumulative distribution function (CDF) of SVS for all channels measured

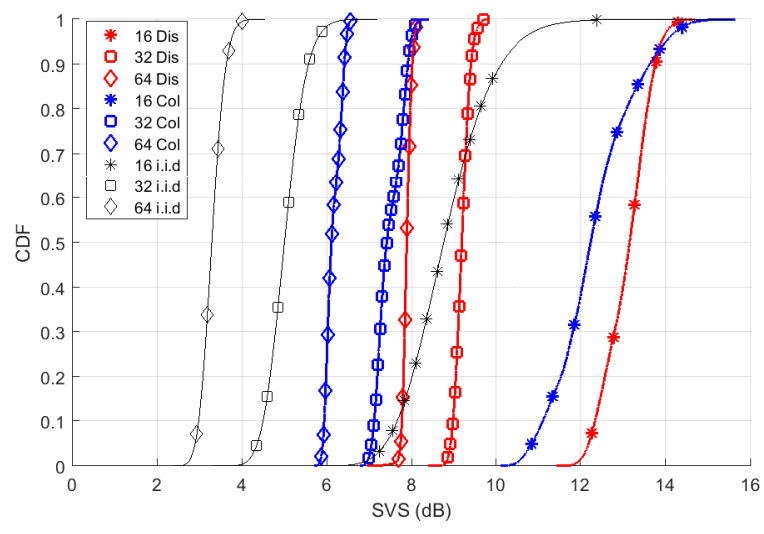

Fig. 6. CDF of SVS w.r.t. different number of active antennas: 16, 32 and 64. An i.i.d Rayleigh channel is included here. CAA outperforms DAAs for the same total number of antenna elements.

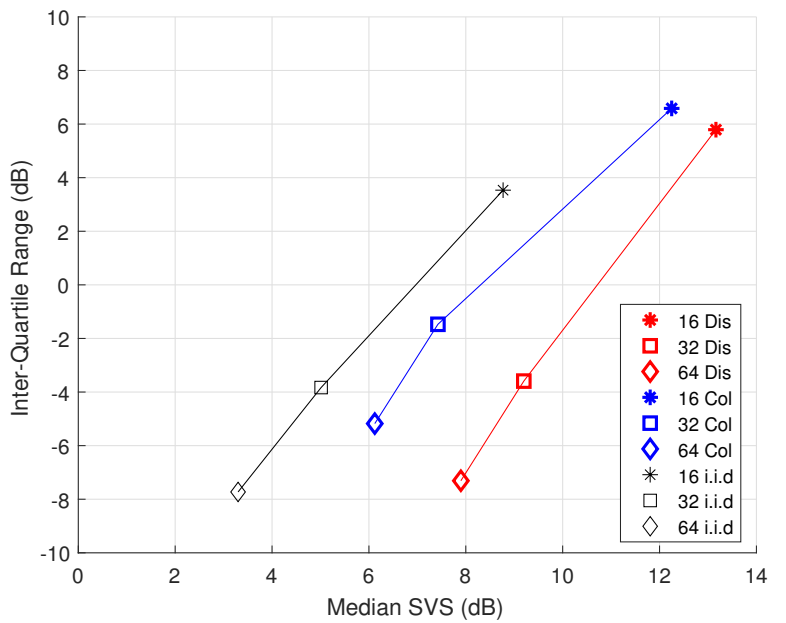

Fig. 7. Median and IQR of SVS values for the same cases as Fig.6

in both scenarios. An i.i.d. channel is also included for comparison. As the number of antennas increases the SVS decreases, so we get more orthogonal channels. Interestingly, 32 antenna CAA outperforms even 64 antenna DAAs case in terms of median SVS. However, the smaller standard deviation in SVS represented here as IQR can be understood as better $\mathrm{CH}$ and addressing the impact of fading. Therefore, SVS of median together with IQR of the SVS is plotted as shown in Fig.7 What is important here is that for the 32 and 64 antenna cases, IQR of DAAs approach that of the i.i.d. channel. Noteworthy there is a trade-off between $\mathrm{CH}$ and channel orthogonality, dependent on the sub-array location in our experiment.

\section{E. User Fairness and Throughput}

We obtained the overall achievable throughput as reported in Table I for each array size and both topologies. The system achieved almost peak throughput for both 64 antenna cases. For 32 antennas, in Table II, we see a larger imbalance between users for the collocated scenario, while the distributed deployment achieves a more homogeneous performance across UEs. This makes sense as the BS is more distributed in the 


\begin{tabular}{|l|c|c|c|c|c|c|c|c|c|c|c|c|}
\hline \multicolumn{10}{|c|}{32 antennas scenario, throughput per UE [Mbps] } \\
\hline & UE 1 & UE 2 & UE 3 & UE 4 & UE 5 & UE 6 & UE 7 & UE 8 & UE 9 & UE 10 & UE 11 & UE 12 \\
\hline Collocated & 17.80 & 14.33 & 18.04 & 17.95 & 18.11 & 17.95 & 16.99 & 16.15 & 18.20 & 18.20 & 16.84 & 10.05 \\
\hline Distributed & 18.20 & 18.20 & 18.19 & 18.02 & 17.97 & 17.69 & 17.70 & 16.88 & 18.21 & 18.22 & 18.21 & 18 \\
\hline
\end{tabular}

TABLE II. 32 antennas scenario, throughput per UE [Mbps]

distributed topology. For the 16 antennas case, both antenna topologies cannot achieve a robust service to all 12 UEs, and all users achieve a low performance. For our measurement scenario, DAAs consistently give a higher throughput for 64 , 32 and 16 active antennas, and reach the maximum throughput of $218.8 \mathrm{Mbits} / \mathrm{sec}$. When the number of antennas is scaled down from 64 to 32 , there is a minor throughput drop for DAAs but the decrease is more pronounced for CAA. From the UL point of view, if we fix the constellation to 16QAM, the best option is 32 antennas in the distributed topology, as we can reach almost similar throughput as that of 64 antennas case and also save half the required number of antennas and frontend hardware. However, we agree it is dangerous to generalize this result to all scenarios and deployments and more field tests are needed to conclude on the most optimal configuration.

\section{CONCLUSION}

Let us go back to the question we tried to answer in this paper, for a specific MaMIMO deployment scenario: how to scale up MaMIMO: should we add more antennas to a given cell, or create multiple smaller and distributed cells that can cooperate? This paper details the scenario and analyzes the performance in terms of throughout, channel hardening, array gain and channel orthogonality for some dense MaMIMO deployment scenarios in a given indoor-to-outdoor NLoS scenario. Specifically, we compare a centralized versus split deployment of a MaMIMO array, using a total of 16, 32 and 64 antenna elements and 12 UE. We focus on a NLoS scenario, as BS and UE are separated by a brick wall, windows, heating elements and a shelf. It is shown that the end throughput result is a combination of multiple complex factors that influence the end performance, as MaMIMO performance depends both on array gain (resulting from the effective number of antennas) and diversity gain (resulting from the Channel Hardening). The results show that increasing the number of antennas improves performance for each topology in a similar way. For our evaluated scenario, a distributed (split array) deployment allows a higher throughput for the same number of antenna elements. The underlying analysis shows that we cannot conclude that the array gain, or effective number of antennas seen by the users is drastically different for both considered topologies. What is seen however, is that the variance across users is decreased when distributing the sub-arrays, i.e., the spread of the received power from each UE is smaller for distributed deployments. This benefits the fairness, and also, in our scenario, the sum throughput in the network. When analyzing the SVS we come to the same conclusions. For our measurements, we cannot conclude that the orthogonality of the channel is improved when distributing the sub-arrays, on the contrary. However, the spread of variation across SVS for multiple channel realisations is improved in the distributed case. While the results are indeed strongly dependent on the exact deployment scenario, we believe that our measurements results allow for some careful early conclusions. First, when the number of antenna elements is large, in a small cell scenario, the topology or location of the antenna elements is not that important. When the channel is good and orthogonal enough, nothing is to be gained by placing the arrays optimally. Second, when the number of UE is large compared to the number of antenna elements (12 versus 32 ), the array topology matters more and the distribution of the antenna's helps to improve the spread of the performance across users). All in all, we believe it is fair to say that the distribution of antennas improves performance. We can however not yet conclude that it is an optimal topology, especially if the extra deployment cost can not be neglected.

\section{ACKNOWLEDGEMENTS}

This work was partially funded by the a Flemish Hercules grant, and by the European Union's Horizon 2020 under grant agreement no. 732174 (ORCA project). Furthermore, the authors would like to thank Prof. L. Van der Perre for discussions related to the measurement setup and analysis.

\section{REFERENCES}

[1] E. G. Larsson, F. Tufvesson, O. Edfors, and T. L. Marzetta, "Massive MIMO for next generation wireless systems," IEEE Commun. Mag., vol. 52, pp. 186-195, Feb. 2014.

[2] X. Gao et al., "Massive MIMO Performance Evaluation Based on Measured Propagation Data," IEEE Trans. Wireless Commun., vol. 14, no. 7, 2015, pp. 3899-3911.

[3] J. Flordelis, X. Gao, G. Dahman, F. Rusek, O. Edfors, and F. Tufvesson, "Spatial separation of closely-spaced users in measured massive multiuser MIMO channels," in Proc. IEEE ICC, June 2015.

[4] M. Gauget et al., "Channel measurements with different antenna array geometries for massive MIMO systems," in Proc. Int. ITG Conf. Syst., Commun. Coding (SCC), Feb. 2015, pp. 1-6.

[5] C.-M. Chen, V. Volskiy, A. Chiumento, L. Van der Perre, G.A. Vandenbosch, S. Pollin, "Exploration of User Separation Capabilities by Distributed Large Antenna Arrays. "Globecom Workshops (GC Wkshps), 2016 IEEE. IEEE, 2016, pp. 1-6.

[6] S. Zhou, M. Zhao, X. Xu, J. Wang, and Y. Yao, "Distributed wireless communication system: A new architecture for future public wireless access," IEEE Commun. Mag., vol. 41, no. 3, pp. 108-113, Mar. 2003.

[7] H. Q. Ngo, A. E. Ashikhmin, H. Yang, E. G. Larsson, and T. L. Marzetta, "Cell-free massive MIMO versus small cells," IEEE Trans. Wireless Commun., 2016, submitted. [Online]. Available: http://arxiv.org/abs/1602.08232

[8] C.-M. Chen, S. Blandino, A. Gaber, C. Desset, A. Bourdoux, L. Van der Perre, and S. Pollin, "Distributed Massive MIMO: A Diversity Combining Method for TDD Reciprocity Calibration," in Proc. Globecom, Dec. 2017.

[9] J. Vieira et al., "A Flexible 100-Antenna Testbed for Massive MIMO," Proc. IEEE Globecom Wksp. -Massive MIMO: From Theory to Practice, 2014.

[10] C.-M. Chen, V. Volskiy, L. Van der Perre, G.A. Vandenbosch, S. Pollin, "Finite large antenna arrays for Massive MIMO: characterisation and system impact," IEEE Transactions on Antennas and Propagation, 2017. 